\title{
LA PRISIÓN Y DESPUÉS \\ VIOLENCIA, REINGRESO Y SITUACIÓN DE CALLE
}

\section{Fiorella Ciapessoni}

\section{Resumen}

Los incidentes de violencia en prisión tienen consecuencias negativas sobre el bienestar físico, psicológico y social de la población privada de libertad, y agravan además los problemas asociados con la vuelta a la vida en comunidad luego de la puesta en libertad. En este artículo se introducen los distintos enfoques sobre la violencia en la vida intramuros y se sistematiza la literatura referida a sus impactos en los procesos de reingreso comunitario. A partir de los argumentos expuestos, se reflexiona sobre las potenciales implicancias de la violencia en prisión en posteriores experiencias de situación de calle para el caso uruguayo. El artículo procura contribuir al diseño de las intervenciones sobre población liberada en situación de exclusión residencial extrema.

Palabras clave: violencia, prisión, reingreso comunitario, situación de calle.

\section{Abstract}

Before and after prison. Violence, re-entry and street homelessness

Incidents of violence in prison have negative consequences on the physical, psychological and social well-being of imprisoned offenders, aggravating also the problems associated with community reentry of released. The paper reviews the different approaches of violence in prison, and introduces key literature on impacts of violence prison on community re-entry. The paper reflects on the potential implications of violence in prison in subsequent experiences of street homelessness in the local context. The article seeks to contribute to the design of social interventions on released offenders experiencing homelessness after prison experiences.

Keywords: violence, prison, community re-entry, street situation.

Fiorella Ciapessoni: Docente e investigadora del Departamento de Sociología, Facultad de Ciencias Sociales, Universidad de la República de Uruguay (UdelaR). Estudiante de doctorado del programa Social Policy y Social Work de la Universidad de York, Reino Unido. Integrante del Núcleo de Análisis de la Criminalidad y la Violencia (NACVI), Facultad de Ciencias Sociales (UdelaR). ORCID iD: https://orcid.org/0000-0002-0447-3935

E-mail: fciape@gmail.com 


\section{Introducción}

La violencia en la prisión es un asunto ampliamente abordado por la literatura internacional. La violencia interpersonal en los establecimientos penitenciarios refiere a conductas o actos violentos (muertes violentas, homicidios, autolesiones físicas, agresiones verbales, abuso sexual, robos de pertenencias) que generan un temor considerable entre los afectados o entre quienes presencian agresiones a terceros, pero que, sin embargo, no plantea desafío alguno para el continuo funcionamiento de la prisión como organización, a diferencia de la violencia colectiva (revueltas, motines) (Bottoms, 1999). No obstante, ese tipo de violencia es un problema grave, con efectos perjudiciales en la salud física y mental de las víctimas y testigos, sus familias y el personal penitenciario (Randol y Campbel, 2017). Distintos estudios coinciden en señalar que el temor generado por vivir en un ambiente penitenciario violento y amenazante es uno de los factores de mayor estrés entre los internos y funcionarios (Toch, 1977, citado en Listwan, Hanley y Colvin, 2012).

Si bien los sucesos de violencia durante el encierro son un asunto arduo de explorar y documentar, y a pesar de que prevalece un subreporte de las experiencias de abuso, las cifras existentes son preocupantes (Bottoms, 1999; McCorkle, 1993). Por otra parte, a pesar de que ha sido bien documentado que la población privada de libertad está expuesta a tasas más altas de violencia que la población general, escasos son los estudios que vinculen la exposición a la violencia o a experiencias de victimización en el entorno carcelario con el proceso de reingreso a la comunidad. Los antecedentes existentes señalan que la primera tiene consecuencias negativas sobre el bienestar psicológico de la población privada de libertad (mayores niveles de estrés, ansiedad y bajo autocontrol) que agravan, además, las dificultades asociadas con la vuelta a la vida en comunidad (abuso de sustancias, falta de soporte familiar, pocas posibilidades de acceder a empleo y alojamiento sostenido) (Listwan, Hanley y Colvin, 2012; Kilpatrick, Saunders y Smith, 2003).

La transición de la prisión a la comunidad es aún más crítica para quienes presentan múltiples episodios de encarcelamiento, problemas de abuso de sustancias, diagnósticos de salud mental graves sin tratamiento o problemas de vivienda previos al ingreso a prisión, cuestiones que, combinadas, pueden resultar en experiencias de situación de calle (Gowan, 2002; Carlen, 1996; Gouvis y Travis, 2004; Herbert, Morenoff y Harding, 2015; Baldry y Maplestone, 2003). 
En Uruguay, las malas condiciones de reclusión y los sucesos de violencia en las cárceles son un asunto preocupante para las agencias internacionales, los actores locales y las organizaciones de derechos humanos. Del mismo modo, las dificultades asociadas al egreso penitenciario se han vuelto temas de creciente preocupación en materia de seguridad pública.

En este marco, el presente trabajo reseña parte de la literatura y estudios empíricos internacionales que discuten los impactos que tiene la violencia durante el encierro en el proceso de reingreso de la población liberada a la comunidad, con especial énfasis en sus posibles implicancias en experiencias de aislamiento social y situación de calle. El artículo se organiza de la siguiente manera: en primer lugar, se revisa la literatura internacional especializada en violencia en el ámbito carcelario, mediante un acercamiento entre los distintos modelos teóricos que buscan explicarla, para, en segundo lugar, introducir la literatura sobre los impactos de la violencia en prisión en el proceso de reingreso comunitario. En tercer lugar, se reflexiona, para el caso uruguayo, sobre las implicancias de las experiencias de victimización en prisión en posteriores experiencias de situación de calle, con el objetivo de contribuir al diseño de intervenciones orientadas a población liberada en situación de exclusión residencial.

\section{Enfoques sobre violencia en prisión}

Existe una vasta literatura que busca explicar las causas de la violencia interpersonal en prisión, con tres enfoques teóricos prevalecientes. El primero se basa en factores individuales y propone que la relación entre ciertos atributos de las personas (edad, raza, experiencias de victimización previas, problemas de abuso de sustancias) e historias personales preexistentes al encierro puede explicar las variaciones en los niveles de violencia en las prisiones (Gaes y McGuire, 1985; Bierie, 2012; Jiang y Fisher-Giorlando, 2002).

Según este enfoque, los varones jóvenes que forman parte de pandillas, tienen experiencias previas de encarcelamiento con antecedentes de violencia en prisión o severos problemas de salud mental tienden a estar más involucrados en sucesos de violencia durante el encierro (DeLisi, Berg y Hochstetler, 2004; Drury y DeLisi, 2011; Rocheleau, 2015). Esta perspectiva, denominada modelo de importación, sostiene que los sucesos de violencia en la cárcel son el resultado de una subcultura externa relacionada con actitudes violentas y trayectorias personales disfuncionales que los internos trasladan a los establecimientos penitenciarios (Irwin y Cressey, 1962; Gaes y McGuire, 1985; Bierie, 2012).

Otra perspectiva, también basada en factores individuales y con un enfoque sociopsicológico, sugiere que las conductas violentas de los individuos encarcelados son producto de la falta de capacidades de los individuos para 
hacer frente a los factores estresantes (problemas, situaciones difíciles) de ese entorno (Blevins, et al., 2010; Toch, 1997, citado en McCorkle, Miethe y Drass, 1995; Toman, Cochran y Bales, 2015). En este sentido, algunos estudios señalan que las probabilidades de inadaptación y comportamiento violento de los individuos durante el encarcelamiento están influenciadas por haber sufrido durante la infancia sucesos de violencia y victimización directa (abuso físico o sexual) ejercidos por adultos conocidos (Meade y Steiner, 2013).

Por el contrario, el enfoque de la privación se basa en factores institucionales como las malas condiciones de reclusión y el hacinamiento, el control administrativo y la severidad en la aplicación de las reglas, la composición del personal penitenciario y la población carcelaria, las relaciones entre estos, la escasa disponibilidad de programas de rehabilitación, entre otros, para explicar la prevalencia de la violencia en las cárceles. En general, estos estudios argumentan que las condiciones desagradables de la prisión llevan a los reclusos a experimentar la coacción, lo que a su vez genera diversos problemas para los reclusos y los sistemas penitenciarios (Liebling y Arnold, 2004, citados en Bierie, 2012; Cao, Zhao y Van Dine, 1997; Camp, et al, 2003; Farrington y Nuttall, 1980; Gaes y McGuire, 1985; Drago, Galbiati y Vertova, 2011).

En los estudios que responden a esta perspectiva se señala que las condiciones opresivas del entorno penitenciario crean una atmósfera que impide la adaptación de la población privada de libertad a la vida en el encierro y provocan frustración, altos niveles de ansiedad y estrés que explican no solo los sucesos de violencia interpersonal y colectiva, sino también las altas tasas de suicidio entre la población carcelaria (Gaes, 1992; Sykes, 1958; Bottoms, 1999; Moos, 1976; Glass y Singer, 1972; Suedfeld, 1977, citados en Huey y McNaulty, 2005). Además, estos estudios muestran que en las prisiones de máxima seguridad la falta de control sobre la autonomía personal, el espacio físico y las rutinas diarias incide en que existan más casos de suicidio y violencia que en las prisiones de seguridad mínima y media (Adams, 1992; Liebling, 1999; Huey y McNulty, 2005).

Otras investigaciones señalan que las condiciones ambientales deficientes, particularmente el hacinamiento, refuerzan las actitudes punitivas (más castigos y de mayor gravedad) hacia los internos por parte del personal penitenciario, como el aislamiento en celdas de castigo, donde es más probable que ocurran suicidios (Huey y McNulty, 2005). Además, características como la alta rotación de personal y las poblaciones transitorias de reclusos son también posibles contribuyentes a la violencia (Camp, et al., 2003; Wooldredge, 1999; Wortley, 2002).

No obstante, la evidencia empírica no es concluyente y algunos estudios señalan que el efecto específico del hacinamiento no puede ser identificado porque se confunde con otras variables del espacio físico como el tamaño de la prisión, la densidad poblacional y la distancia interpersonal (Camp, 
et al., 2003; Gaes y McGuire, 1985). A partir de la crítica del estrés como variable explicativa de los disturbios en prisión, algunos estudios (McCorkle, Miethe y Drass, 1995) se centran en medidas de privación relativa, como, por ejemplo, los cambios en los niveles de privación, y la violencia colectiva es explicada a partir de las discrepancias entre lo que los individuos esperan y lo que efectivamente obtienen (Martin y Zimmerman, 1990, citado en McCorkle, Miethe y Drass, 1995).

Más allá de algunas excepciones, existe consenso sobre que el estrés provocado por las condiciones de hacinamiento y las luchas por los escasos recursos, sumados a la pérdida de autonomía, generan un ambiente que impide la adaptación de los internos a la vida en reclusión y aumenta los sucesos de violencia y las probabilidades de suicidio (Gaes, 1992; Huey y McNulty, 2005; Bottoms, 1999; Rocheleau, 2015).

La tercera perspectiva, una variante de carácter mesoestructural del enfoque institucional denominada perspectiva situacional, resalta la importancia de los factores de la situación de la prisión como precipitadores de la violencia (Wortley, 2002; Rocheleau, 2015; Tartaro y Levy, 2007; Bottoms, 1999; Griffin y Hepburn, 2013). Las interacciones entre los internos, el personal y el ambiente (Randol y Campbell, 2017); los sucesos de violencia, que aumentan el riesgo de lesiones y estrés, que, a su vez, conducen a índices altos de ausentismo, insatisfacción laboral y rotación de personal penitenciario (Cullen, et al., 1993); los cambios en la reglas y las fallas en la gestión del espacio penitenciario; y eventos que ocurren fuera de la prisión (por ejemplo, disturbios o hechos de violencia en otras prisiones) pueden afectar el clima de la prisión y, con ello, la respuesta de la población privada de libertad en cuanto al comportamiento violento (Endler y Magnusson, 1976; Goodstein, MacKenzie y Shotland, 1984; Steinke, 1991, citados en Meade y Steiner, 2013).

Buena parte de los estudios con este enfoque muestra que los internos se comportan de manera diferente según cómo sean sus interacciones con los demás, el tipo de prisión y la estructura carcelaria en la que se encuentren, las formas de afrontar los conflictos, las respuestas institucionales a estos y los innumerables factores situacionales (Bottoms, 1999; Edgar, O'Donnell y Martin, 2003, citado en Rocheleau, 2015). En esta línea, Bottoms (1999) señala que la incidencia de actos de violencia interpersonal dentro de la prisión está influenciada por las características de los internos, pero también por aspectos ambientales y por la continua interacción dinámica del personal penitenciario y el contexto físico y social de la prisión.

\section{Violencia en prisión y reingreso comunitario}

Una vez revisada la parte de la literatura que retrata los potenciales impactos de las experiencias de violencia y victimización durante el encarcelamiento, 
es oportuno abordar las implicancias que tienen estas experiencias en la población liberada una vez que ocurre el regreso a la comunidad.

A modo de ejemplo, una investigación de Boxer, Middlemass y Delorenzo (2009) basada en el modelo de adaptación buscó determinar el efecto de la violencia durante el encarcelamiento, tanto para víctimas como para testigos, en el ajuste psicológico de liberados violentos y no violentos luego del egreso. ${ }^{1}$ El estudio señala que, a pesar de que los liberados presentaban al ingresar a prisión elevadas actitudes antisociales, haber estado expuestos a sucesos violentos durante el encierro agravó sus problemas de comportamiento, lo que se manifestó en agresión o conducta antisocial y en síntomas como angustia emocional, depresión y ansiedad. Sostiene, además, que estos problemas psicosociales tienen un efecto duradero que se extiende más allá de la liberación.

Otro estudio empírico (Listwan, et al., 2010) buscó examinar los efectos psicológicos de sufrir coacción directa e indirecta durante el encarcelamiento, así como el impacto del apoyo social en la salida en libertad en más de 1.600 ofensores recientemente liberados. ${ }^{2} \mathrm{El}$ trabajo buscó dar cuenta de los efectos de ciertos factores de carácter individual y otros de índole estructural en el bienestar psicológico de los individuos puestos en libertad. La hipótesis central es que la coerción (incluida la victimización) experimentada en el interior de un recinto penitenciario aumenta los síntomas de estrés postraumático, mientras que el apoyo social los reduce (Cullen, 1994) y modera los efectos de percibir la prisión como un ambiente estresante, amenazante y coercitivo. Cuanto más amplias sean las fuentes de apoyo social, menos probable será que la población reclusa sufra estrés postraumático, lo que repercutirá en su bienestar psicológico y, como consecuencia, en las posibilidades de atravesar un proceso de reinserción satisfactorio (Listwan, et al., 2010).

Los resultados de esa investigación señalan que las experiencias de victimización se asocian positivamente con síntomas de trauma que incluyen ansiedad, depresión, disociación, trauma posabuso, trastornos del sueño y problemas sexuales, sobre todo en la población de mayor edad. El estudio subraya también que la victimización directa (medida a través un listado de cogniciones postraumáticas) tiene un impacto negativo sobre la percepción

1 La idea de ajuste psicológico refiere a anomalías de salud mental entre las que se incluyen aspectos físicos, emocionales, cognitivos y sociales: depresión, ansiedad, equilibrio emocional, autoestima, adaptación al medioambiente, conductas violentas y ruptura de normas, uso de estrategias de afrontamiento frente a situaciones estresantes, etcétera. (Achenbah y Rescorla, 1987).

2 Los autores retoman la definición de Colvin (2000) sobre coerción directa e indirecta, entendiendo por la primera el uso de la fuerza, las amenazas y la intimidación y/o la eliminación o amenaza de la eliminación de apoyo material y emocional. Un ejemplo destacado de coerción directa es ser víctima física (ser golpeado o amenazado con golpizas) y materialmente (ser víctima de robos). La coerción indirecta consiste en presenciar la victimización de otros y vivir en lo que se percibe como un entorno amenazador en el que el miedo y la intimidación se convierten en aspectos predominantes (Listwan, et al., 2010). 
positiva de sí mismo, la identidad y la confianza y la capacidad de sentirse seguro con otros (Foa y Ehlers, 1999).

Por último, un proyecto de investigación más exhaustivo (Listwan, Hanley y Colvin, 2012) explora el impacto de sucesos de victimización emocional, física o sexual en una muestra de 1.600 personas recientemente liberadas residentes en casas de medio camino (centros de transición de estadía de mediano plazo). Algunos de los resultados del estudio señalan que casi el total de los liberados encuestados (97,9\%) presenció durante el encarcelamiento robos, agresiones físicas o violencia sexual. En particular, el $82 \%$ fue testigo de actos de robo, el $92 \%$ de agresiones físicas y el $95 \%$ de agresiones emocionales o verbales. Con respecto a la victimización sexual, 305 encuestados $(19,7 \%)$ indicaron que habían presenciado la coacción sexual de un individuo por otro y el 12,1\% indicó que había visto una violación. El estudio señala también que quienes informaron haber sido víctimas eran más propensos a ser caucásicos, jóvenes y sin hijos, miembros de pandillas, haber participado de peleas, haber sido puestos en celda de aislamiento y haber sido diagnosticados con problemas de salud mental. Contrariamente a lo que podría esperarse, la investigación señala que las víctimas tenían más probabilidades de haber participado en programas de tratamiento durante la prisión y, además, quienes habían participado en ellos presentaban más síntomas de trauma que los no participantes (Listwan, Hanley y Colvin, 2012). Sin embargo, los autores afirman que probablemente el trauma haya llevado a los individuos a buscar tratamiento y no que este último los haya empujado al sufrimiento psicológico (Gavrilovic, Schutzwohl, Fazel y Priebe, 2005).

En relación con los resultados de reingreso, cerca del $50 \%$ de los encuestados volvió a prisión y el $17 \%$ fue arrestado por delito violento. El análisis multivariado del arresto revela que el haber presenciado victimización sexual en prisión es un factor importante para predecir el arresto durante la vida en libertad. En el marco del estudio, quienes tenían más probabilidades de volver a ser arrestados resultaron ser los ofensores liberados más jóvenes, que habían experimentado una victimización violenta directa en prisión, con un diagnóstico de problema de salud mental y un mayor número de condenas por delitos graves, participantes en programas de tratamiento y desempleados (Listwan, Hanley y Colvin, 2012).

Como vemos, el impacto de haber experimentado o presenciado violencia física o sexual en prisión se relaciona con una multiplicidad de dificultades y problemas psicológicos y comportamentales no solo durante la estadía en prisión, sino también después del egreso (Listwan, et al., 2013; Meade y Steiner, 2013). A las graves consecuencias de la victimización durante el encierro se suman, durante el proceso de reingreso a la comunidad, las complejidades de restablecer la vida después de la cárcel: encontrar un lugar 
para vivir, retomar los vínculos familiares y cercanos positivos, encontrar un empleo, etcétera (Travis, 2005; Petersilia, 2001 y 2011).

El proceso de reingreso está moldeado por la complejidad y la interacción de factores como la historia personal del ofensor, sus vivencias durante el encierro, la naturaleza del/los delito/s cometido/s, el abuso de sustancias, las habilidades laborales, la salud mental y física, la relación con la familia y con pares criminales, las actitudes personales y creencias sobre su vuelta a la comunidad, las oportunidades concretas de apoyo para que su vida sea diferente esta vez, las necesidades específicas pero cambiantes que se presentan inmediatamente a la liberación, a mediano y largo plazo, entre otros factores (Visher y Travis, 2011).

Quienes tienen problemas de abuso de sustancias psicoactivas, escasos años de escolarización y múltiples sentencias desde edades tempranas presentan los mayores déficits para enfrentar un proceso de reingreso sostenido (Travis, Chincotta y Solomon, 2003; Listwan, Hanley y Colvin, 2012). Además, los servicios pospenitenciarios tienen efectos limitados para reducir de manera significativa la reincidencia delictiva, ya que las fallas de los programas de reinserción aparecen antes de la salida de la población ofensora y los efectos colaterales del encarcelamiento (sanciones civiles que exceden el sistema penal y que afectan los derechos y deberes ciudadanos de las personas liberadas) siguen siendo las principales barreras para la reintegración (Petersilia, 2011; Uggen, Manza y Thompson, 2006).

Sin la supervisión adecuada de y en la comunidad es poco probable que la población liberada reciba el apoyo social necesario para lograr un proceso de reingreso social sostenido y exitoso (Listwan, Hanley y Colvin, 2012). Por otra parte, como vimos, los trastornos depresivos, de estrés postraumático $\mathrm{y}$ de ansiedad, el abuso de sustancias y una variedad de desventajas relacionadas con la salud y el bienestar psicológico emergen como algunas de las consecuencias de haber sufrido agresiones físicas repetidas y sostenidas en prisión (Listwan, Hanley y Colvin, 2012).

Conjuntamente, la evidencia muestra que las fallas en los programas de rehabilitación y reinserción afectan las tasas de reincidencia delictiva y el reencarcelamiento, pero también los niveles de situación de calle, lo que hace que las personas liberadas sean vulnerables a episodios de inestabilidad residencial y a la falta de vivienda segura y estable durante el proceso de reinserción (Williams, et al., 2012; Dore, 2015; Gowan, 2002; Brown, 2006; Metraux y Culhane, 2002; Baldry, et al., 2006; Mayock y Sheridan, 2013; Dyb, 2009). Además, buena parte de los ofensores liberados es cada vez más vulnerable a experiencias de situación de calle como resultado del aumento de la población carcelaria y el consiguiente aumento del número de personas que salen de la cárcel con recursos y servicios limitados para facilitar su reingreso a la comunidad (Gouvis y Travis, 2004; La Vigne, et al., 2003; Cho, 2004; Herbert, et al., 2015). 
Los estudios prospectivos han encontrado que las experiencias de situación de calle se producen de manera desproporcionada después de la salida de hogares de cuidado, de tratamiento y de prisión, y afectan a entre una décima y una tercera parte de la población liberada de esas instituciones (Metraux, et al., 2007; Pleace, 2016). Más aún, el sistema penal ha sido identificado como un factor que genera experiencias de inestabilidad residencial, aislamiento y situación de calle en gran parte de la población liberada (Herbert, et al., 2015).

Quienes son liberados y no tienen donde vivir vuelven a las comunidades o barrios criminógenos o a soluciones de alojamiento inseguras de corto y mediano plazo (hostales, refugios nocturnos) que agravan el proceso de transición a la comunidad, ya sea por el reencuentro con pares delincuentes o porque la inestabilidad de esas soluciones habitacionales coloca a los individuos en riesgo inmediato de fracaso (Kushel, et al., 2005; Steiner, et al., 2012, citados en Lutze, Rosky y Hamilton, 2014; Sapouna, et al., 2015; Dore, 2015). Además, por lo general, un gran número de expresos tenía problemas habitacionales antes del encarcelamiento, lo que empeora la transición de la prisión a la comunidad y aumenta el riesgo de regresar a la prisión (Gouvis, 2004; Meatraux y Culhane, 2006).

Quienes egresan de la prisión sufren, además, las consecuencias colaterales del encierro, como la falta de autonomía, el estrés y el daño emocional, la pérdida de autoestima, la falta de relaciones sociales, los problemas de salud y el estigma, lo que podría resultar en experiencias de aislamiento social y situación de calle (Gouvis, 2004; Herbert, et al., 2015; Arditti, 2003; Hagan y Foster, 2012; Petersilia, 2001; Baldry y Maplestone, 2003). Las experiencias de sucesos de violencia en prisión afectan a mediano y largo plazo la salud mental de la población privada de libertad, por la exposición constante al estrés, la intimidación y los abusos (Flannery, 2006), pero, además, quienes al egresar deben vivir a la intemperie son potenciales víctimas de abuso y violencia en el espacio público (Huey, 2010) y, como consecuencia, quedan en una situación de doble victimización que disminuye las posibilidades de reincorporarse exitosamente a la sociedad.

Las personas en situación de calle padecen múltiples y persistentes desventajas entrelazadas (abuso de sustancias, problemas de salud mental, algún grado de involucramiento en delitos menores, débiles o nulos vínculos sociales) que dan como resultado una "exclusión profunda y persistente" (Bramley, et al., 2015; Duncan y Corner, 2012) y la experiencia de altas tasas de hostigamiento, riesgo de vida y victimización (Huey, 2007; Lee y Schreck, 2005; Hagan y McCarthy, 1997). En especial, quienes presentan la más severa combinación de múltiples y graves desventajas son aquellos que tienen entre 29 y 49 años, especialmente quienes están en la década de los treinta (Bramley, et al., 2015). 
El reingreso o retorno a la comunidad es un hecho que sucederá en algún momento para la amplia mayoría de las personas encarceladas (excepto para quienes fallecen mientras están encarcelados) (Travis, 2005). Ese proceso de retorno será más o menos satisfactorio según las características individuales, las relaciones familiares, el contexto comunitario, los servicios sociales y los recursos institucionales disponibles (Visher y Travis, 2011; Petersilia, 2011). La evidencia muestra que las experiencias de victimización en la cárcel agravan el proceso de reingreso, intensifican los síntomas de sufrimiento psicológico y aumentan las posibilidades de recaer en comportamientos disruptivos (Listwan, Hanley y Colvin, 2012; Drago, et al., 2011). Los lazos de confianza y el apoyo social actúan como amortiguadores del impacto del trauma provocado por los sucesos de violencia y demás eventos estresantes (Listwan, et al., 2010), tienen resultados positivos en la autopercepción y contribuyen a disminuir la alienación social (Paterline y Petersen, 1999, citados en Listwan, et al., 2010).

\section{Panorama en Uruguay}

Uruguay enfrenta un panorama complejo en relación con los niveles de personas en situación de calle (en intemperie y en centros nocturnos), el crecimiento de la población carcelaria y las políticas de reinserción de esas poblaciones. Por un lado, desde mediados del 2000 el problema de la situación de calle es considerado "una problemática extremadamente compleja y multicausal, donde la vulnerabilidad social se refleja, no solo en las condiciones socioeconómicas, sino que se asocia a otros componentes como: consumo problemático de sustancias psicoactivas, alcoholismo, trastornos mentales crónicos, determinando un debilitamiento en la vinculación con las redes sociales, pérdida de habilidades laborales, etc.” (Mides, 2016b).

Los relevamientos oficiales muestran que la población en calle y en centros nocturnos ha aumentado de manera sostenida desde el primer censo realizado en 2006. Los datos oficiales de 2016 para Montevideo exhiben un aumento del 53\% en comparación con el censo de 2011, con un total de 1.651 personas en refugios y calle durante la noche del relevamiento (Mides, 2016a). En comparación con el relevamiento de 2011, en Montevideo, el día del censo, hubo un $26 \%$ más de personas en intemperie (556 individuos) y un $59 \%$ más de usuarios de centros nocturnos (1.095 personas) (Mides, 2016a). Específicamente, para quienes habitan a la intemperie se realizó por primera vez un censo que relevó información esencial sobre las particularidades de esa población.

El informe del censo destaca que el $94 \%$ son varones adultos solteros y el $64 \%$ tiene menos de 40 años, mientras que el 33\% se ubica en el tramo etario de 31 a 40 años (Mides, 2016a). La ruptura de vínculos es el motivo desencadenante — según los encuestados — de su situación de calle, 
mencionado por más del $55 \%$, seguido por adicciones $(30 \%)$ e insuficiencia de ingresos (20\%). En menor medida se señalan experiencias de violencia $(5,6 \%)$, salida de privación de libertad $(2,5 \%)$ y el padecimiento de una patología de salud mental (2\%) (Mides, 2016a). No obstante, el 55\% de los encuestados declara mantener algún vínculo o relación con familiares (madre y hermanos en su mayoría) que no se encuentran en situación de calle. Entre quienes mantienen vínculos con familiares, más del $50 \%$ se contactó en la última semana y si se amplía el período consultado a menos de un mes, se alcanza al 70\% de los casos (Mides, 2016a). En relación con el tiempo en situación de calle, el $80 \%$ declara haber dormido los siete días de la última semana en la calle (Mides, 2016a) y un poco más del 50\% ha estado en esa situación por menos de un año desde la última experiencia de calle.

Además, ocho de cada diez encuestados señalan haber tenido en algún momento contacto con centros nocturnos, Ley de Faltas y equipo móvil del Ministerio de Desarrollo Social (Mides); cuatro de cada diez personas a la intemperie y dos de cada diez en refugios declaran haber estado privados de libertad en algún momento de sus vidas; tres de cada diez expresan haber estado institucionalizados en el Instituto del Niño y Adolescente del Uruguay (INAU); dos de cada diez, haber estado internados en una institución psiquiátrica. En síntesis, seis de cada diez individuos que habitan a la intemperie han vivido al menos un episodio de institucionalización (Mides, 2016a).

Por otro lado, en lo que hace al sistema penal y al delito, Uruguay presenta un panorama arduo. El país tiene la tasa de prisión más alta de la región, estimada en 306 por cada 100.000 habitantes (Prison Studies, 2017), lo que lo posiciona "entre los primeros 30 países con la mayor cantidad de presos en el mundo [...], en el $8^{\circ}$ lugar de Sudamérica y el $79^{\circ}$ en el mundo en términos de hacinamiento" (Comisionado Parlamentario para el Sistema Penitenciario, 2016; IPCS, 2015, citado en González, Trajtenberg y Rojido, 2015). El crecimiento de la población carcelaria durante los últimos veinte años se ha combinado con un crecimiento sostenido de los delitos más violentos, en particular en Montevideo y el área metropolitana. Además, según datos oficiales, la reincidencia ha crecido de manera constante entre 2000 y 2014, pasando del $49 \%$ al 59\%. Estas cifras, junto con el sentimiento de inseguridad pública, han resultado ser un tema de preocupación principal para la sociedad uruguaya (Paternain y Rico, 2012; Vigna, 2011).

La población penitenciaria está compuesta mayoritariamente por varones (94\%) solteros y con escasos años de escolarización; casi el 70\% tienen menos de 35 años (Juanche y Palummo, 2012; Vigna, 2012). El 4,5\% de los ofensores varones declaró estar en situación de calle antes de ingresar a la prisión (Vigna, 2012).

Existe consenso sobre que se han efectuado avances importantes en materia penitenciaria, como la creación del Instituto Nacional de 
Rehabilitación (INR), la implementación de la escala de riesgo Offender Assessment System (OASyS) para clasificar la situación de las personas que ingresan al sistema penitenciario y adecuar las medidas de rehabilitación de acuerdo con las situaciones específicas en diversas áreas relevadas, una mayor presencia de funcionarios penitenciarios, la paulatina reducción del personal policial y un mayor control a las prácticas ilegales arraigadas, y continuos esfuerzos en términos de reducción del hacinamiento y de protección y cumplimiento de los derechos humanos de los internos.

No obstante, persisten severos problemas, tales como déficits de atención en salud mental de la población privada de libertad (pacientes psiquiátricos sin tratamiento o con tratamiento inadecuado), consumo problemático de sustancias, prevalencia de ciertas enfermedades contagiosas (tuberculosis), denuncias de abusos y violaciones a los derechos básicos (mala alimentación, por ejemplo), escasísimo tiempo fuera de las celdas, ocio compulsivo, inexistencia de programas sociales, familiares y comunitarios y escasa preparación para el egreso y apoyo pospenitenciario. Además, en algunas cárceles persisten niveles críticos de hacinamiento (Comisionado Parlamentario para el Sistema Penitenciario; 2016, 2017; Garcé, 2017).

"El hacinamiento es uno de los factores que obstaculiza el desarrollo de los programas de rehabilitación, si el número de presos va más allá del sistema, el colapso de programas y actividades genera violencia interna". (Comisionado Parlamentario para el Sistema Penitenciario, 2016)

En lo relativo a la violencia en las cárceles uruguayas, estas presentan, en comparación con la región, una situación altamente desfavorable y preocupante debido a los altos niveles de violencia intragrupal y las altas tasas de suicidios, homicidios y muertes violentas. Según Garcé (2017), casi la mitad de las lesiones en población privada de libertad son producto de actos intencionales con el fin de agredir físicamente (golpes, cortes, quemaduras), amenazas, cobros extorsivos ("peajes") o de abuso sexual (no denunciado por miedo o amenazas) con el fin de obtener alguna ventaja material. El país alcanzó en 2016 el número más alto de homicidios entre personas privadas de libertad, con una tasa de 155 cada 100.000 , y entre 2012 y 2016 la tasa de suicidios se multiplicó por cuatro (Garcé, 2017; Comisionado Parlamentario para el Sistema Penitenciario, 2016). Las muertes violentas no son la excepción, y en 2015 fallecieron en forma violenta 43 internos (Garcé, 2017).

En materia de reinserción social, si bien hay avances, sobre todo en relación con los programas de empleo y educación, el país aún no cuenta con una política integral de reingreso de la población privada de libertad, lo que afecta profundamente las condiciones de vida de la población ofensora al ser liberada y limita sus posibilidades de lograr una liberación exitosa y una reintegración comunitaria satisfactoria (Comisionado Parlamentario para el Sistema Penitenciario, 2016; Naciones Unidas, 2009; Folle y Vigna, 2015; 
Camaño, 2011). La Dirección Nacional de Apoyo al Liberado (DINALI), dependiente del Ministerio del Interior, trabaja en pos de apoyar la reinserción social de la población liberada a través de mecanismos de intervención que promuevan la autonomía y el apoyo personal de quienes tuvieron experiencias penitenciarias, para evitar así la reincidencia. Coordina acciones con otras instituciones por ejemplo el Mides, los municipios, la Administración de los Servicios de Salud del Estado (ASSE) y diversos programas sociales e interviene en materia habitacional ofreciendo alojamiento transitorio (no más de sesenta días en un período de cinco años después del egreso) a las personas liberadas del sistema penitenciario que no tengan a donde ir (Comisionado Parlamentario para el Sistema Penitenciario, 2016).

No obstante, como vimos antes, es reconocido que en buena parte de los grandes establecimientos penitenciarios el estado crítico de hacinamiento, el ocio compulsivo, el acceso limitado a los servicios de salud, la inadecuación de los espacios físicos, las experiencias de violencia o la exposición a abusos o malos tratos (en su mayoría no denunciados), la falta de actividades de capacitación socioeducativa, sumados a la ausencia de una política de reinserción integral, parecerían afectar en profundidad las circunstancias de vida de la población ofensora que regresa a la comunidad.

Un trabajo reciente muestra que para el conjunto de personas de entre 18 y 36 años que habitaban espacios públicos la noche en que fueron censadas (Mides, 2016a) existe una asociación positiva entre experiencias de consumo abusivo con problemas de salud mental, así como una asociación directa entre los problemas en la órbita penal y el dormir a la intemperie (Ciapessoni, 2018). También para ese grupo etario, las desventajas estructurales adquieren mayor relevancia en el análisis de correlación: la exclusión educativa se asociada directamente a la ruptura de vínculos y, a través de la exclusión del mercado de trabajo, está también asociada a dormir a la intemperie. Para el conjunto de los individuos, la situación de calle está asociada de manera directa con la ruptura de vínculos $\mathrm{y}$, en menor grado, con haber tenido problemas judiciales (Ciapessoni, 2018). El trabajo enfatiza en la urgencia de atender las necesidades y problemas específicos de quienes están en la franja de los treinta años y presentan características que los colocan en un extremo de la exclusión asociada a la situación de intemperie (nunca hicieron uso de centros nocturnos, han estado privados de libertad, fueron retirados de la calle por la Ley de Faltas) (Ciapessoni, 2018).

En este marco, el acceso a una vivienda segura y estable aparece como uno de los temas pendientes para alcanzar un proceso de reinserción satisfactorio para quienes son liberados (Ciapessoni y Vigna, 2018) e intervenir estratégicamente en la prevención de las experiencias de calle parece ser una de las acciones más urgentes en materia de reinserción social. La atención a la salud mental, el adecuado tratamiento de las adicciones, el egreso planificado del sistema penal, coordinado con otros organismos, y 
evitar la recriminalización de personas con trayectorias de exclusión severa son algunos de los desafíos en la atención a la población liberada en situación de calle.

\section{Reflexiones finales}

En primer lugar, distintas investigaciones internacionales que vinculan el impacto de las experiencias de victimización en el reingreso a la vida comunitaria muestran los efectos negativos que la exposición a hechos de violencia y eventos estresantes tiene en la población liberada. De acuerdo con los estudios empíricos reseñados, haber sido víctima o testigo de violencia grave en prisión está relacionado con una multiplicidad de dificultades y problemas psicológicos y comportamentales que se mantienen después del egreso (Listwan, Hanley y Colvin, 2012; Meade y Steiner, 2013). Las víctimas de violencia a menudo sufren mayores niveles de estrés, ansiedad, malestar emocional y una variedad de desventajas relacionadas con la salud en general (Briere y Jordan, 2004; Krupnick, et al., 2004; Sommers y Buschur, 2004; Stein, et al., 2004, citados en Listwan, et al., 2010).

Haber sufrido eventos de violencia física o sexual o haber estado expuesto a ellos impacta desfavorablemente en el proceso de transición de la prisión a la vida en libertad. Los estudios señalan, además, que experimentar eventos traumáticos (amenazas, abusos físicos) en prisión provoca emociones negativas, como miedo intenso y estrés constante, que pueden llevar a problemas significativos y de largo plazo en términos de salud mental y comportamiento y pueden derivar en experiencias de aislamiento social y situación de calle. Los lazos de confianza actúan en parte como amortiguadores de los impactos del trauma generado por la victimización (Cullen, et al., 1993; Listwan, et al., 2010), mientras que la pérdida y el debilitamiento de los vínculos sociales positivos han sido identificados como factores que afectan la reintegración de la población liberada a la comunidad (Loucks, 2007; Roman y Travis, 2004; Hickey, 2002; Travis, et al., 2001; Maruna, 2001; Walker, et al., 2014; Farrall, 2002).

La evidencia empírica señala que las personas liberadas tienden a ser cada vez más vulnerables a experiencias de situación de calle, como resultado del aumento de la población carcelaria y el consiguiente aumento en el número de personas que salen de la cárcel con recursos y servicios limitados para el reingreso (Gouvis y Travis, 2004; La Vigne, et al., 2004; Cho, 2004; Herbert, et al., 2015). La literatura sobre reingreso señala que el acceso a una solución habitacional inmediata a la liberación y sostenible en el tiempo, que contemple las necesidades específicas del individuo liberado, es un componente esencial para la reintegración exitosa en la comunidad, con un impacto considerable en las oportunidades de encontrar empleo, la gestión de las rutinas diarias, la construcción de capital social y la reducción de la 
inestabilidad y la precariedad residencial y las posibilidades de reincidencia (Dore, 2015; Harper y Chitty, 2005; Sapouna, et al., 2011; Cullen, et al., 1999, citado en Lutze, Rosky y Hamilton, 2014; Allen y Barkley, 2002).

Para quienes han sufrido victimización en prisión, las posibilidades de tener una reinserción eficaz parecen ser aún menos. Si bien las maneras de afrontar la victimización dependen de la gravedad del hecho en sí, de las respuestas institucionales, de la calidad y la estabilidad de los vínculos de la persona victimizada y de su capacidad personal para afrontar el suceso (Rocheleau, 2015), haber sido testigo o víctima de violencia - sobre todo de abuso sexual - intensifica las dificultades psicológicas (ansiedad, estrés postraumático, depresión, etcétera) de las personas liberadas.

Por último, en relación con el caso uruguayo, creemos pertinente que poder contar, mediante futuros relevamientos, con información sobre experiencias o exposición a distintos tipos de violencia durante el encierro de quienes egresan del sistema penitenciario y están en situación de calle es central para un mejor diseño de los programas encargados de la reinserción de esta población. Esta información permitirá apreciar de forma más compleja y profunda cómo la exposición constante y la acumulación de vivencias traumáticas en prisión pueden asociarse a desventajas psicosociales que resultan en experiencias de calle luego del egreso. Finalmente, relevar información sobre experiencias de victimización ocurridas desde edades tempranas, que impactan de manera desfavorable en la salud mental de las personas, parecería también ser central para un mejor conocimiento de los procesos que subyacen a las trayectorias o experiencias de encarcelamiento y exclusión residencial severa. 


\section{Referencias bibliográficas}

Achenbach, T. M. y C. Edelbrock (1987). Manual for the Youth Self: Report and Profile. Burlington: University of Vermont, Department of Psychiatry.

Adams, K. (1992). Adjusting to prison life. Crime and Justice, 16, pp. 275-359.

Allen, C. y D. Barkley (2002). Housing for Offenders: The Role of 'Understanding Relationships'. Supporting People, 49(4), pp. 267-276.

pp. 275-359. Chicago: University of Chicago Press.

Arditti, J. (2003). Locked doors and glass walls: Family visiting at a local jail. Journal of Loss and Trauma, 8(2), pp. 1-23.

Baldry, E. y P. Maplestone (2003). Prisoners post-release homelessness and lack of social integration. Current Issues in Criminology, 15(2), pp. 155-169.

Baldry, E.; D. McDonnell; P. Maplestone y M. Peeters (2006). Ex-Prisoners, Homelessness and the State in Australia. The Australian and New Zealand Journal of Criminology, 39(1), pp. 21-33.

Bierie, D. M. (2012). Is tougher better? The impact of physical prison conditions on inmate violence. International Journal of Offender Therapy and Comparative Criminology, 56(3), pp. 338-355.

Blake, M. R. y C. M. Campbell (2017). Macro-Correlates of Inmate Violence: The Importance of Programming in Prison Order. The Prison Journal, 97(4), pp. 451-474.

Blevins, K. R.; S. J. Listwan; F. T. Cullen; y C. L. Jonson (2010). A General Strain Theory of Prison Violence and Misconduct: An Integrated Model of Inmate Behavior. Journal of Contemporary Criminal Justice, 26(2), pp. 148-166.

Bramley, G; S. Fitzpatrick; J. Edwards; D. Ford; S. Johnsen; F. Sosenko y D. Watkins, D. (2015). Hard Edges: mapping severe and multiple disadvantage in England. London: Lankelly Chase Foundation.

Bottoms, A. (1999). Interpersonal Violence and Social Order in Prisons. Crime and Justice, 26, pp. 205-281.

Bowker, L. (2012). An Essay on Prison Violence. Pensilvania: SAGE.

Boxer, P.; K. Middlemass y T. Delorenzo (2009). Exposure to violent crime during incarceration: Effects on psychological adjustment following release. Criminal Justice and Behavior, 36(8), pp. 793-807.

Brown, K. (2006). Homelessness and prisoner re-entry. Nueva York: Council of State Governments. 
Butler, M. y S. Maruna (2009). The Impact of Disrespect on Prisoners' Aggression: Outcomes of Experimentally Inducing Violence Supportive Cognitions. Psychology, Crime and Law, 15(2-3), pp. 235-250.

Camaño, D. (2011). Aportes a la reforma del Código Penal y el Código Procesal Penal. Montevideo: PNUD-MI.

Camp, S. D.; G. G. Gaes; N. P. Langan y W. G. Saylor (2003). The influence of prisons on inmate misconduct: A multilevel investigation. Justice Quarterly, 20(3), pp. 501-533.

Cao, L.; J. Zhao y S. Van Dine (1997). Prison disciplinary tickets: A test of the deprivation and importation models. Journal of Criminal Justice, 25(2), pp. 103-113.

Carlen, P. (1996). Jigsaw: A political criminology of youth homelessness. Buckingham y Bristol, PA: Open University Press.

Cho, R. (2004). Putting the pieces back together: Overcoming fragmentation to prevent post-incarceration homelessness. Nueva York: Corporation for Supportive Housing.

Ciapessoni, F. y A. Vigna (2018). El rol de la vivienda en el proceso de desistimiento delictivo. El Uruguay desde la Sociología, XVI, pp. 259-278.

Ciapessoni, F. (2018, en prensa). Situación de calle en Montevideo: necesidades $y$ desafios. Montevideo: FCS-IM.

Colvin, M. (2000). Crime and coercion: An integrated theory of chronic criminality. Nueva York: Palgrave Macmillan

Comisionado Parlamentario para el Sistema Penitenciario (2016). Informe anual 2016. Parlamento del Uruguay.

Comisionado Parlamentario para el Sistema Penitenciario (2017). Informe anual 2017. Parlamento del Uruguay.

Comisionado Parlamentario para el Sistema Penitenciario (2018). Informe especial sobre el Módulo 8 de la Unidad para Personas Privadas de Libertad $n .^{\circ} 4$ del Instituto Nacional de Rehabilitación (COMCAR). Parlamento del Uruguay.

Cullen, F. T. (1994). Social support as an organizing concept for criminology: Presidential address to the Academy of Criminal Justice Sciences. Justice Quarterly, 11(4), pp. 527-559. 
Cullen, F. T.; E. Latessa; V. Burton y L. Lombardo (1993). The correctional orientation of prison wardens: Is the rehabilitative ideal supported? Criminology, 31(1), pp. 69-92.

Dammert, L. y L. Zúñiga (2008). La cárcel: problemas y desafíos para las Américas. Santiago, Chile: FLACSO.

Day, J. C.; J. R. Brauer y H. D. Butler (2015). Coercion and social support behind bars: testing an integrated theory of misconduct and resistance in U.S. prisons. Criminal Justice and Behavior, 42(2), pp. 133-155.

DeLisi, M.; M. T. Berg. y A. Hochstetler (2004). Gang members, career criminals and prison violence: Further specification of the importation model of inmate behavior. Criminal Justice Studies, 17(4), pp. 369-383.

Dore, E. (2015). Prison leavers and homelessness. Insight, 29, pp. 3-19.

Drago, F.; R. Galbiati y P. Vertova (2011). Prison conditions and recidivism. American Law and Economics Review, 13(1), pp. 103-130.

Drury, A. J. y M. DeLisi (2011). Gangkill: An exploratory empirical assessment of gang membership, homicide offending, and prison misconduct. Crime $y$ Delinquency, 57(1), pp. 130-146.

Duncan M. y J. Corner (2012). Severe and Multiple Disadvantage-A review of keytexts. Londres: Lankelly Chase Foundation.

Dyb, E. (2009). Imprisonment: A Major Gateway to Homelessness. Housing Studies, 24(6), pp. 809-824.

Edgar, K.; I. O'Donnell; y C. Martin (2003). Prison violence: The dynamics of conflict, fearand power. Portland, OR: Willan Publishing.

Farrall, S. (2002): Rethinking what works with offenders on probation, social context and desistance from crime. Current Issues in Criminal Justice, 16:(1), pp. 119-121.

Farrington, D. y C. P. Nuttall (1980). Prison Size, Overcrowding, Prison Violence, and Recidivism. Journal of Criminal Justice, 8(4), pp. 221-231.

Flannery, D. J. (2006). Violence and mental health in everyday life: Prevention and intervention strategies for children and adolescents. Walnut Creek, CA: AltaMira Press.

Foa, E. B.; A. Ehlers; D. M. Clark; D. F. Tolin; y S. M. Orsillo (1999). The posttraumatic cognitions inventory (PTCI): Development and validation. Psychological Assessment, 11(3), pp. 303-314. 
Folle, M. y A. Vigna (comps.). Cárceles en el Uruguay en el siglo XXI. Montevideo: CSIC- UdelaR.

Gaes, G. (1992). The effects of overcrowding in prison. Crime and Justice, 6, pp. $95-146$.

Gaes, G. y W. McGuire (1985). Prison Violence: the contribution of crowding versus other determinants of prison assault rates. Journal of research in crime and delinquency, 22(1), pp. 41-65.

Garcé, A. (2017). Privación de libertad y reinserción social en Uruguay. Montevideo: CERES-CAF.

Gavrilovic, J. J.; M. Schutzwohl; M. Fazel; y S. Priebe (2005). Who seeks treatment after a traumatic event and who does not? A review of findings on mental health service utilization. Journal of Traumatic Stress, 18(6), pp. 595-605.

Gendreau, P.; C. Goggin y F. T. Cullen (1992). The Effects of Prison Sentences on Recidivism. Ottawa: Public Works and Government Services Canada.

Gouvis, C. (2004). A Roof is not enough: Successful Prisoner Reintegration Requires Experimentation and Collaboration. Reaction Essay, 3(2), pp. 161-168.

Gouvis, C. y J. Travis (2004). Taking Stock: Housing, Homelessness, and Prisoner Reentry. Final Report prepared for The Fannie Mae Foundation Urban Institute. Justice Policy Center US.

Gowan, T. (2002). The nexus Homelessness and incarceration in two American cities. Etnography, 3(4), pp. 500-534.

Griffin, M. L. y J. R. Hepburn (2013). Inmate misconduct and the institutional capacity for control. Criminal Justice and Behavior, 40(3), pp.270-288.

Hagan, J. y B. McCarthy (1997). Mean Streets: Youth Crime and Homelessness. Nueva York: Cambridge University Press.

Hagan, J. y Foster, H. (2012). Intergenerational Educational Effects of Mass Imprisonment in America. Sociology of Education, 85(3), pp. 259-286.

Harper, G. y C. Chitty (eds.) (2005). The impact of corrections on re-offending: a review of 'what works'. Londres: Home Office Research.

Herbert, C.; J. D. Morenoff y D. J. Harding (2015). Homelessness and Housing Insecurity among Former Prisoners, the Russell Sage Foundation. Journal of the Social Sciences, 1(2), pp. 44-79. 
Hickey, C. (2002). Crime \& Homelessness. Dublín: Focus Ireland \& PACE.

Huey, L. (2007). Negotiating Demands: The Politics of Skid Row Policing in Edinburgh, San Francisco and Vancouver. Toronto: University of Toronto Press.

Huey, L. (2010). False security or greater social inclusion? Exploring perceptions of CCTV use in public and private spaces accessed by the homeless. The British Journal of Sociology, 61(1), pp. 63-82.

Huey, L. (2012). Invisible Victims: Homelessness and the Growing Security Gap. Toronto, Ontario: University of Toronto Press.

Huey, M. y T. McNaulty (2005). Institutional Conditions and Prison Suicide: Conditional Effects of Deprivation and Overcrowding. The Prison Journal, 85(4), pp. 490-514.

Irwin, J. y R. D. Cressey (1962). Thieves, Convicts and the Inmate Culture. Social Problems, 10(2), pp. 142-15.

Jiang, S. y M. Fisher-Giorlando (2002). Inmate misconduct: A test of the deprivation, importation and situational models. The Prison Journal, 82(3), pp. 335-368.

Juanche, A. y J. Palummo (eds) (2012). Hacia una política de Estado en privación de libertad: Diálogo, recomendaciones y propuestas. Montevideo: SERPAJ.

Kilpatrick, D. G.; B. E. Saunders y D. W. Smith (2013). Youth Victimization: Prevalence and Implications. Washington: National Institute of Justice.

La Vigne, N. G.; C. A. Mamalian; J. Travis; y C. Visher (2003). A portrait of prisoner Reentry in Illinois. Washington, DC: The Urban Institute.

Lee, B. A. y C. J. Schreck (2005). Danger on the Streets Marginality and Victimization Among Homeless People. American Behavioral Scientist, 48(8), pp. 1055-1081.

Liebling, A. (1999). Prison suicide and prisoner coping. En M. Tonry y J. Petersillia (eds.), Prisons. Chicago: University of Chicago Press, pp. 283-359.

Listwan, S.; M. Colvin; D. Hanley y D. Flannery (2010). Victimization, Social Support and Psychological Well-Being: A Study of Recently Released Prisoners. Criminal Justice and Behavior, 37(10), 1140-1159.

Listwan, S.; D. Hanley y M. Colvin (2012). The Prison Experience and Reentry: Examining the Impact of Victimization on Coming Home, Final Report. Ohio: National Institute of Justice. 
Liebling, A. y S. Maruna (2005). The Effects of Imprisonment. Cullompton, Devon: Willan Publishing.

Loucks, N. (2007). Housing Needs of Prisoners and Ex-Prisoners. Glasgow: The Robertson Trust.

Lutze, F. E; J. W. Rosky y Z. K. Hamilton (2014). Homelessness and Reentry. A Multisite Outcome Evaluation of Washington State's Reentry Housing Program for High Risk Offenders. Criminal Justice and Behavior, 41(4), pp. 471-491.

Maguire, M. y P. Raynor (2006). How the resettlement of prisoners promotes desistance from crime: Or does it? Criminology \& Criminal Justice, 6(1), pp. 19-38.

Maruna, S. (2001). Making good: How ex-convicts reform and rebuild their lives. Washington: American Psychological Association.

Maruna, S. (2011). Reentry as a rite of passage. Punishment y Society, 13(1), pp. 3-28. Mayock, P. y S. Sheridan(2013). At Home' in Prison? Women and the HomelessnessIncarceration Nexus. Irish Probation Journal, 10, pp. 118-140.

McCorkle, R. C. (1993). Fear of victimization and symptoms of psychopathology among prison inmates. Journal of Offender Rehabilitation, 19(1-2), pp. 27-42.

McCorkle, R.; T. Miethe y K. Drass (1995). The roots of prison violence: a test of the deprivation, management, and "not-so-total" institution models. Crime y Delinquency, 41(3), pp. 317-331.

Meade, B. y B. Steiner (2013). The effects of exposure to violence on prisoner maladjustment. Criminal Justice and Behavior, 40(11), pp. 1228-1249.

Metraux, S. y D. P. Culhane (2002). Homeless Shelter Use and Re-incarceration Following Prison Release: Assessing the Risk. Filadelfia: University of Pennsylvania.

Metraux, S. y D. Culhane (2006). Recent incarceration history among a sheltered homeless population. Crime and Delinquency, 52(3), pp. 504-517.

Metraux, S.; C. Roman; y R. S. Cho (2007). Incarceration and Homelessness. En D. Dennis, G. Locke y J. Khadduri (eds.). Understanding Homelessness: The 2007 National Symposium on Homelessness Research. Washington: US Department of Housing \& Urban Development.

Mides (2016a). Presentación de resultados del Censo de Población en Situación de Calle. Montevideo: Mides. 
Mides (2016b). Llamado a organizaciones de la sociedad civil (OSC) y/o cooperativas de trabajo para presentar propuestas de trabajo para la gestión de centros de atención en la ciudad de Montevideo e interior en el marco del Programa Calle. Pliego de Bases y Condiciones particulares. Montevideo: Mides.

Morris, R. G. y J. L. Worrall (2014). Prison architecture and inmate misconduct: A multilevel assessment. Crime y Delinquency, 60(7), pp. 1083-1109.

Morris, R. G.; M. Carriaga; B. Diamond; N. Piquero y A. Piquero (2012). Does prison strain lead to prison misbehavior? An application of general strain theory to inmate misconduct. Journal of Criminal Justice, 40(3), pp. 194-201.

Naciones Unidas (2009). Report of the Special Rapporteur on torture and other cruel, inhuman or degrading treatment or punishment, Manfred Nowak. A/HRC/10/44/Add.3, disponible en: <https://www.refworld.org/ docid/49a55fea2.html> [acceso 30/05/2019].

Paternain, R. y A. Rico (2012). Uruguay: Inseguridad, delito y Estado, Montevideo: CSIC-TRILCE.

Petersilia, J. (2001). Prisoner Reentry: Public Safety and Reintegration Challenges. The Prison Journal, 81(3), pp.360-375.

Petersilia, J. (2011). Reentry: Saving Offenders from a Life in Crime. En F. T. Cullen y C. L. Jonson, Correctional Theory: Context and consequences. Londres: Sage Publications.

Pleace, N. (2016). Researching Homelessness in Europe: Theoretical Perspectives. European Journal of Homelessness, 10(3), pp. 19-44.

Pleace, N. y D. P. Culhane (2016). Better than Cure? Testing the case for Enhancing Prevention of Single Homelessness in England. Londres: Crisis.

Randol, B. M. y C. M. Campbell (2017). Macro-Correlates of Inmate Violence: The Importance of Programming in Prison Order. The Prison Journal, 97(4), pp. 451-474.

Ricciardelli, R. (2014). Coping strategies: Investigating how male prisoners manage the threat of victimization in federal prisons. The Prison Journal, 94(4), pp. 411-434.

Rocheleau, A. M. (2013). An empirical exploration of the "pains of imprisonment" and the level of prison misconduct and violence. Criminal Justice Review, 38(3), pp. 354-374. 
Rocheleau, A. M. (2015). Ways of Coping and Involvement in Prison Violence. International Journal of Offender Therapy and Comparative Criminology, 59(4), pp. 359-383.

Roman, C. y J. Travis (2004). Taking Stock: Housing, Homelessness and Prisoner Reentry. Final Report prepared for The Fannie Mae Foundation. Washington: Urban Institute Justice Policy Center.

Sapouna, M; C. Bisset; A. M. Conlong; y B. Matthews (2015). What Works to Reduce Reoffending: A Summary of the Evidence. Edimburgo: Scottish Government.

Sapouna, M; C. Bisset; y A. M. Conlong (2011). What Works to Reduce Reoffending: A Summary of the Evidence. Edimburgo: Justice Analytical Services Scottish Government.

Steiner, B. y B. Meade (2016). Assessing the Link between Exposure to a Violent Prison Context and Inmate Maladjustment. Journal of Contemporary Criminal Justice, 32(4), pp. 328-356.

Tartaro, C. y M. P. Levy (2007). Density, inmate assaults, and direct supervision jails. Criminal Justice, 18(4), pp. 395-417.

Toman, E.; J. Cochran y W. Bales (2015). The implications of sentence length for inmate adjustment to prison life. Journal of Criminal Justice, 43(6), pp. 510-521.

Travis, J. (2000). But They All Come Back: Rethinking Prisoner Reentry. Papers from the Executive Sessions on Sentencing and Corrections, 7, National Institute of Justice.

Travis, J. (2005). But They All Come Back: Facing the Challenges of Prisoner Reentry. Washington DC: The Urban Institute Press.

Travis, J.; A. Solomon; y M. Waul (2001). From Prison to Home: the Dimensions and Consequences of Prisoner Reentry. Washington: The Urban Institute.

Travis, J.; E. M. Chincotta; y A. L. Solomon (2003). Families left behind: the hidden cost of incarceration and Reentry. Washington: The Urban Institute.

Uggen, C.; J. Manza y M. Thompson (2006). Citizenship, Democracy and the Civic Reintegration of Criminal Offenders. Annals of the American Academy of Political and Social Science, 605(1), pp. 281-310. 
Vigna, A. (2011). Persistencia y abandono del mundo del delito: diferencias de género en los procesos de desistimiento. Tesis de Maestría en Sociología. Facultad de Ciencias Sociales, Universidad de la República, Montevideo.

Vigna, A. (2012). Análisis de datos del I Censo Nacional de Reclusos desde una perspectiva de género y derechos humanos. Informe final. Montevideo: MIINR-ONU Mujeres.

Visher, C. A y J. Travis (2011). Life on the Outside: Returning Home after Incarceration. The Prison Journal, 91(3), pp. 102S-119S.

Walker, A; L. Hempel; N. Prabha Unnithan; y M. R. Pogrebin (2014). Parole Reentry and Social Capital: The Centrality of Homelessness. Journal of Poverty, 18(3), pp. 315-334.

Williams, K.; J. Poyser; y K. Hopkins (2012). Accommodation, Homelessness and Reoffending of Prisoners: Results from the Surveying Prisoner Crime Reduction (SPCR) Survey. Londres: Ministry of Justice.

Wooldredge, J. (1999). Inmate experiences and psychological well-being. Criminal Justice and Behavior, 26(2), pp. 235-250.

Wortley, R. (2002). Situational Prison Control: Crime Prevention in Correctional Institutions. Cambridge: Cambridge University Press.

\section{Contribución de autoría}

El trabajo en su totalidad fue realizado por Fiorella Ciapessoni. 